Approved for public release;

distribution is unlimited.

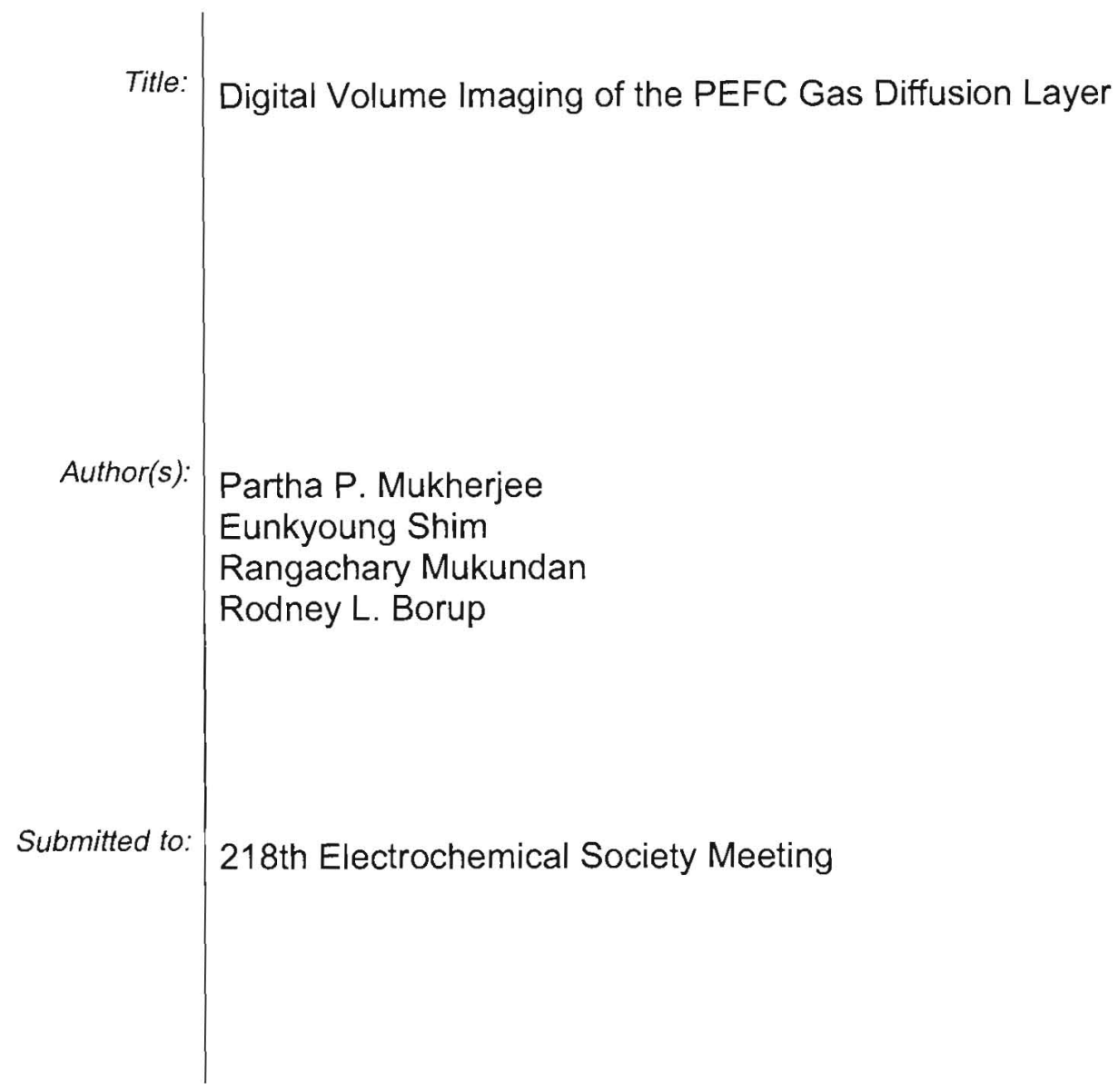

Partha P. Mukherjee

Eunkyoung Shim

Rangachary Mukund

Rodney L. Borup

\section{8th Electrochemical Society Meeting}

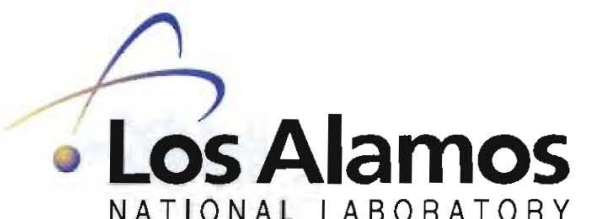

Los Alamos National Laboratory, an affirmative action/equal opportunity employer, is operated by the University of California for the U.S Department of Energy under contract W-7405-ENG-36. By acceptance of this article, the publisher recognizes that the U.S. Government retains a nonexclusive, royalty-free license to publish or reproduce the published form of this contribution, or to allow others to do so, for U.S. Government purposes. Los Alamos National Laboratory requests that the publisher identify this article as work performed under the auspices of the U.S. Department of Energy. Los Alamos National Laboratory strongly supports academic freedom and a researcher's right to publish; as an institution, however, the Laboratory does not endorse the viewpoint of a publication or guarantee its technical correctness. 


\title{
Digital Volume Imaging of the PEFC Gas Diffusion Layer
}

\author{
Partha P. Mukherjee, ${ }^{\mathrm{a}, \mathrm{c}}$ Eunkyoung Shim, ${ }^{\mathrm{b}}$ Rangachary Mukundan, ${ }^{\mathrm{a}}$ and Rodney L. Borup ${ }^{\mathrm{a}}$ \\ ${ }^{a}$ Los Alamos National Laboratory, Los Alamos, NM 87545, USA \\ 'The Nonwovens Institute, North Carolina State University, Raleigh, NC 27695, USA \\ 'Present address: Oak Ridge National Laboratory, Oak Ridge, TN 37831, USA
}

The gas diffusion layer (GDL) plays a key role in the overall performance/durability of a polymer electrolyte fuel cell (PEFC). Of profound importance, especially in the context of water management and flooding phenomena, is the influence of the underlying pore morphology and wetting characteristics of the GDL microstructure. In this article, we present the digital volumetric imaging (DVI) technique in order to generate the 3-D carbon paper GDL microstructure. The internal pore structure and the local microstructural variations in terms of fiber alignment and fiber/binder distributions are investigated using the several 3-D thin sections of the sample obtained from DVI.

\section{Introduction}

Characterization of transport phenomena in the polymer electrolyte fuel cell (PEFC) gas diffusion layer (GDL) has received significant attention in recent years. The contribution of the GDL, especially on the cathode side, to the mass transport loss originating from suboptimal liquid water transport and flooding phenomena is critical to the overall cell performance/durability limitation and water management $(1,2)$. The pore morphology and wetting characteristics of the GDL profoundly influence the underlying flooding dynamics (3-5).

The multi-faceted functionality of a GDL includes reactant distribution, liquid water transport, electron transport, heat conduction and mechanical support to the membraneelectrode-assembly. Carbon-fiber based porous materials, namely non-woven carbon paper and woven carbon cloth, with thickness $\sim 200-300 \mu \mathrm{m}$, have received wide acceptance as materials of choice for the PEFC GDL owing to high porosity $(\sim 70 \%$ or higher), permeability and good electrical/thermal conductivity. Mathias et al. (6) provided a comprehensive overview of the GDL structure and functions.

Detailed knowledge of the underlying porous microstructure is of paramount importance to the fundamental understanding of the transport characteristics in the GDL. Recent efforts in the GDL microstructure generation include stochastic simulation method and experimental imaging. Schulz and co-workers (7) were the first to develop a stochastic simulation technique to reconstruct 3-D realization of the non-woven carbon paper GDL based on structural inputs, namely fiber diameter, fiber orientation and porosity which can be obtained either directly from the fabrication specifications or indirectly from the SEM (scanning electron microscope) micrographs or by experimental techniques. The stochastic reconstruction technique is described as a Poisson line process with one-parametric directional distribution where the fibers are realized as circular cylinders with a given diameter and the directional distribution provides inplane/through-plane anisotropy in the reconstructed GDL microstructure (7). In another effort, Thiedmann et al. (8) reported a stochastic reconstruction method, which is similar 
in spirit to the method by Schulz et al. (7), to generate 3-D non-woven carbon paper GDL microstructures. This model relies on stacking thin sections of fiber layers with each fiber approximated by a straight cylinder. The thin sections of the GDL are represented by planar random line tessellations, which are built by intersecting lines located at random in the material plane (8). Three-dimensional experimental imaging of the PEFC GDL microstructure based on X-Ray computed tomography has been reported in the literature. Notable works on X-Ray microtomography based 3-D GDL microstructure reconstruction include Garzon and co-workers (9), Ostadi et al. (10,11) Becker et al. $(12,13)$. In another recent effort, Berenjov et al. (14) demonstrated an inexpensive optical reconstruction of the carbon paper GDL structure using a standard bright-field microscope.

In this work, we present the $3-\mathrm{D}$ reconstruction of non-woven carbon paper GDL microstructures using digital volumetric imaging (DVI), which is a fluorescent imaging technique $(15,16)$.

\section{Experimental Approach}

\section{Methodology}

DVI is based on serial sectioning of the sample and is a fluorescent imaging technique that can produce several serial sections directly from the sample block automatically and autonomously without operator involvement. Samples are prepared by first fluorescent dyeing of the fibers, dehydration, and embedding the samples in a polymer matrix. Image acquisition is performed by automated slicing of the polymer matrix and taking microscopic images of the cut surface on the sample block. These serial images are volume-rendered to obtain high-fidelity 3-D images. Serial sections can be further exported to other software programs to obtain relevant information pertaining to the microstructure characteristics. The spatial resolution of the images ranges from $0.44 \mu \mathrm{m}$ to $4.44 \mu \mathrm{m}$ depending upon the size of the sample and objective used. In this work, we report the use of the DVI microimager ${ }^{\circledR}$ system and the techniques developed for investigating the 3-D microstructure of a carbon paper GDL substrate without a microporous layer (MPL). Unlike the non-invasive X-Ray tomography method, the DVI technique is a destructive imaging method.

\section{Data Acquisition and Analysis}

The carbon paper sample is impregnated with the embedding media that was prepared according to the following procedure:

$15.0 \mathrm{~g}$ of Vinylcyclohexene dioxide (VCD) and $9 \mathrm{~g}$ of Propyleneglycoldiglycidyle ether (DER), 39g of Nonenylsuccinic anhydride (NS) were mixed with the overhead stirrer (Heidolph Mechanical Overhead Stirrer, RZR 2020) and 7.2g of Sudan Black B (Acros, CAS\# 4197-25-5) to achieve opacity of the media. $672 \mu 1$ of Dimethylethanolamine (DMAE) was added into the mixture. It was stirred until the mixture became homogeneous and centrifuged for 15 minutes at $5000 \mathrm{rpm}$ to remove coagulated Sudan black particles. Sample stripes of $0.4 \mathrm{~mm} \times 5 \mathrm{~cm}$ size were cut and placed into the DVI sample mold. The sample mold was filled with the embedding media. The sample was centrifuged for 1 hour at $3500 \mathrm{rpm}$ to ensure complete infiltration and was cured overnight in the oven at $70^{\circ} \mathrm{C}$.

The acquisition scheme of the 3-D volumetric images with DVI microimager ${ }^{(B)}$ is shown in Fig. 1. The prepared sample block was placed in the motorized image stage and cut by a diamond knife with the thickness of $0.44 \mu \mathrm{m}$. The surface of the sample block 
was captured with the camera through fluorescent optics. Cutting and image capture were automatically repeated until the number of cycle reached a preset value of 1000 sections. The 20x objectives and UV fluorescent filter cube (XF13-2 filter cube, Omega Optical) were used. Captured sectioned images were used to produce image datasets through a serious of image processing step including inverting and background subtraction to reduce noises. The obtained data sets were further visualized and analyzed with RESView ${ }^{\mathrm{TM}}$ software (Microscience Group Inc.) package.

In the acquired data set, the imaging $\mathrm{Y}$-axis corresponds to the thickness or throughplane direction. The $\mathrm{Z}$-axis corresponds to the sectioning direction. Therefore, the $\mathrm{XZ}$ planes correspond to the planes more or less parallel to the carbon paper surface.

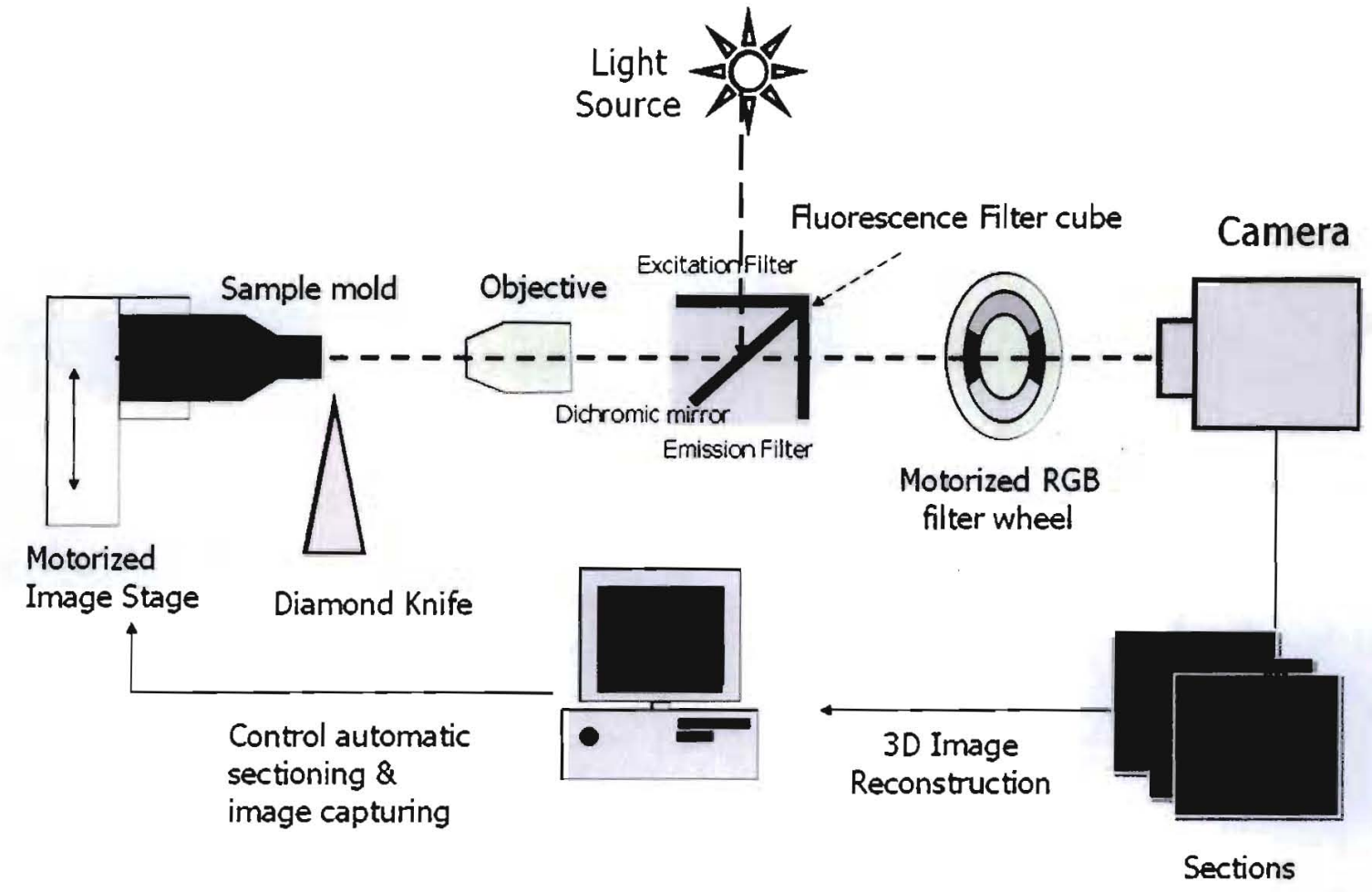

Figure 1: Schematic of the DVI image acquisition setup.

\section{Results and Discussions}

In this section, we present representative results from the DVI reconstruction of a carbon paper GDL microstructure.

Figure 2 shows the SEM image of the GDL sample investigated in this work. The high magnification image, Fig. 2(b), exhibits the binder between the fiber entangles which not only covers the fibers but also blocks the pores.

Figure 3 shows the 3-D volumetric image of the GDL sample obtained using the Resview ${ }^{\circledR}$ software and DVI microimager ${ }^{\circledR}$. The 3-D image is reconstructed using the serial section dataset and consists of $(1000 \times 1000 \times 550)$ voxels, which corresponds to $(440 \mu \mathrm{m} \times 440 \mu \mathrm{m} \times 242 \mu \mathrm{m})$ physical dimensions. From the $3-\mathrm{D}$ image, the complex fiber/binder morphology is evident. 


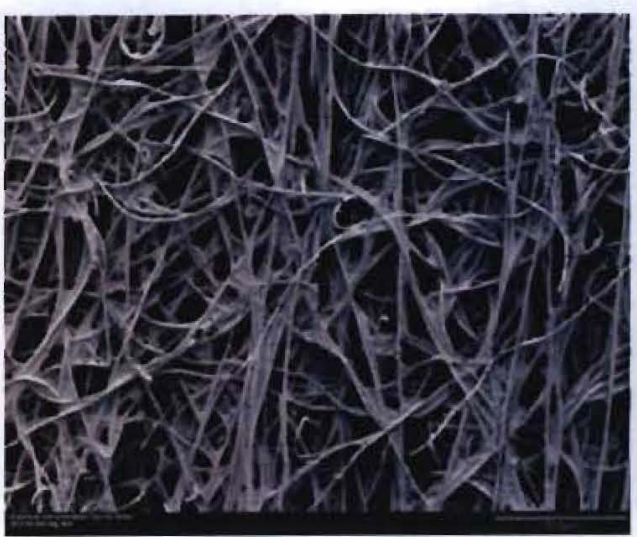

(a)

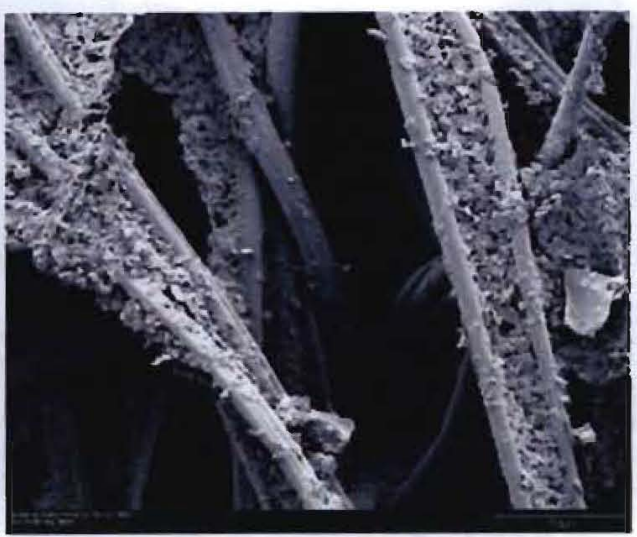

(b)

Figure 2: SEM images of the GDL sample: (a) $50 \times$ (b) $500 \times$ magnification.

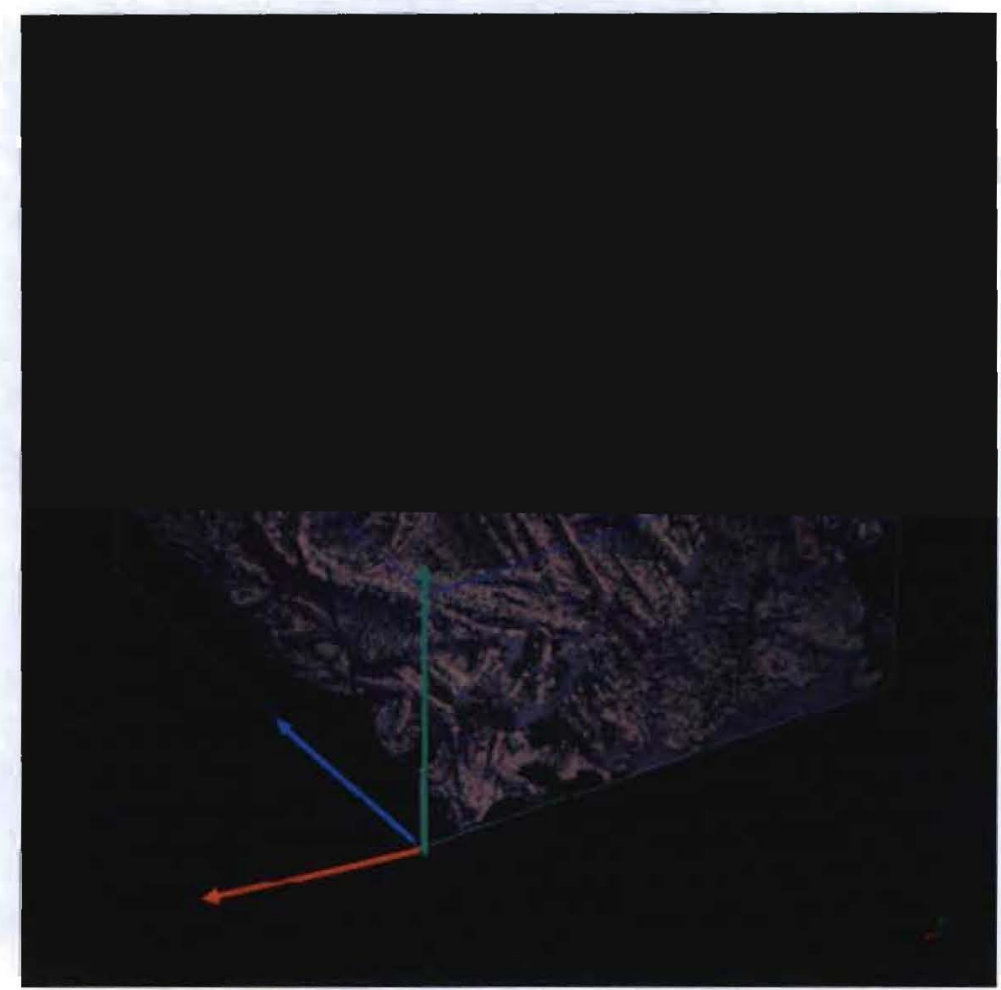

Figure 3: 3-D reconstructed volumetric image of the GDL sample. Red, green and blue arrows correspond to the $\mathrm{X}, \mathrm{Y}$, and $\mathrm{Z}$ axes respectively.

In order to probe the internal pore structure and microstructural variation, a series of thin 3-D sections perpendicular to the through-plane ( $Y$ axis) direction were exported at different $\mathrm{Y}$ positions. These sections are referred to as the $3-\mathrm{D} \mathrm{Y}$ sections. Figure 4 schematically illustrates the schematics of the procedure of exporting the 3-D Y sections, each with size $(440 \mu \mathrm{m} \times 440 \mu \mathrm{m} \times 22 \mu \mathrm{m})$. Figure 5 shows several $3-D$ Y sections along the thickness of the GDL sample. From Fig. 5, it is evident that most fibers are oriented in the Z-direction while only a small fraction of the fibers are oriented in the X direction. The fiber alignment trend agrees well with the SEM observation. It is also important to note that binder distribution varies significantly through the sample thickness. Higher binder content is observed at $\mathrm{Y}=22 \mu \mathrm{m}$, where solid volume fraction (SVF), including fiber and binder, reaches over $40 \%$. At $Y=154 \mu \mathrm{m}$, some fiber surfaces are exposed and 
the binder is predominantly located at the fiber cross-links. The overall binder distribution tends to follow the fiber distribution.

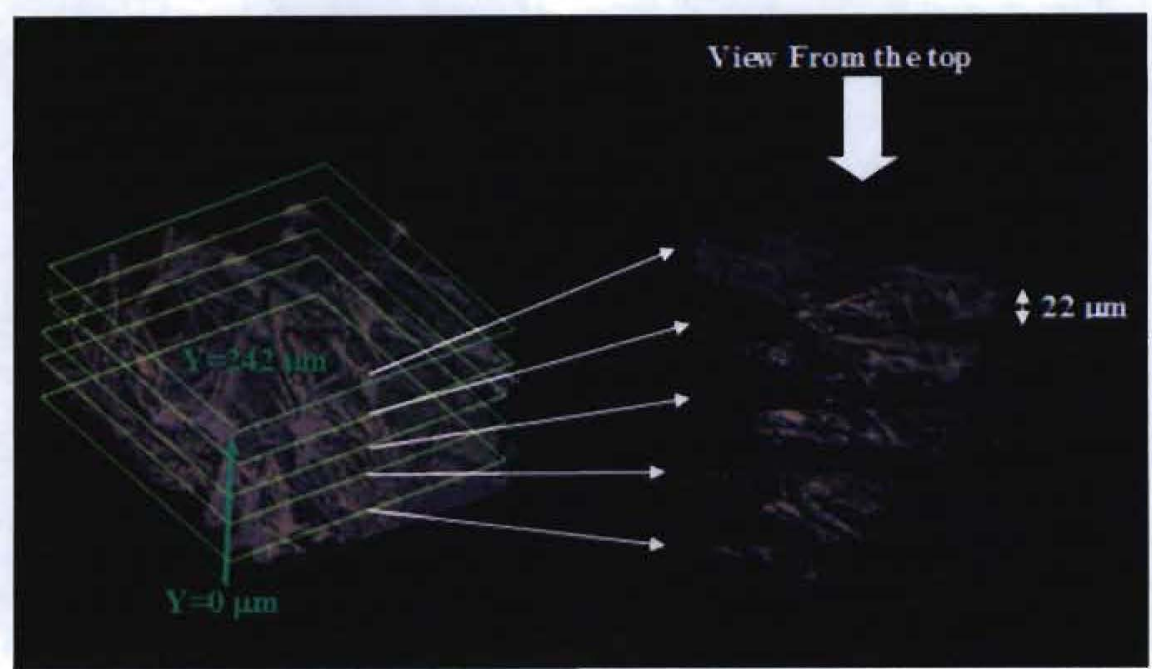

Figure 4: Schematic illustration of 3-D Y section imaging.
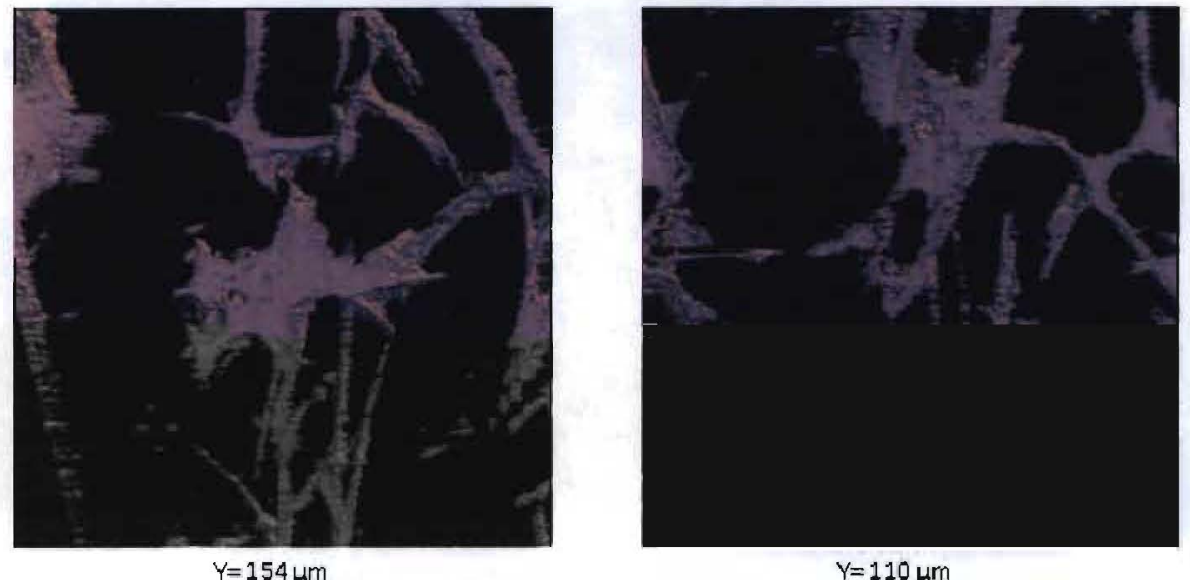

$Y=110 \mu \mathrm{n}$

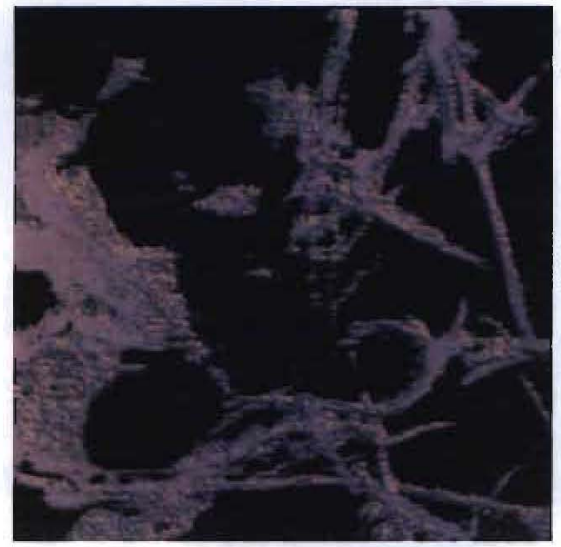

$Y=66 \mu \mathrm{m}$

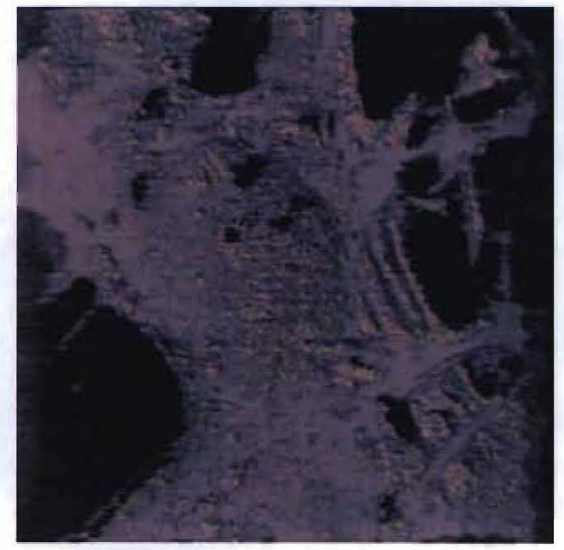

$\mathrm{Y}=22 \mu \mathrm{m}$

Figure 5: Top views of several 3-D Y thin sections along the sample thickness showing the fiber/binder distributions.

Similarly, 3-D thin sections were exported along the $\mathrm{X}$ and $\mathrm{Z}$ directions in order the reveal the internal pore morphology variation, fiber alignment and fiber/binder relative distribution with respect to the through-plane direction. Figures 6 and 7 show the 3-D thin sections along the $\mathrm{X}$ and $\mathrm{Z}$ directions, respectively. Majority of the fibers are 
oriented parallel to the $\mathrm{XZ}$ plane (sample surface plane). However, some fibers, especially in the $Z$ sections, are found to be preferentially oriented in the thickness direction. These 3-D thin sections further suggest that the fiber alignment and binder distribution would significantly influence the through-plane tortuosity of the sample.

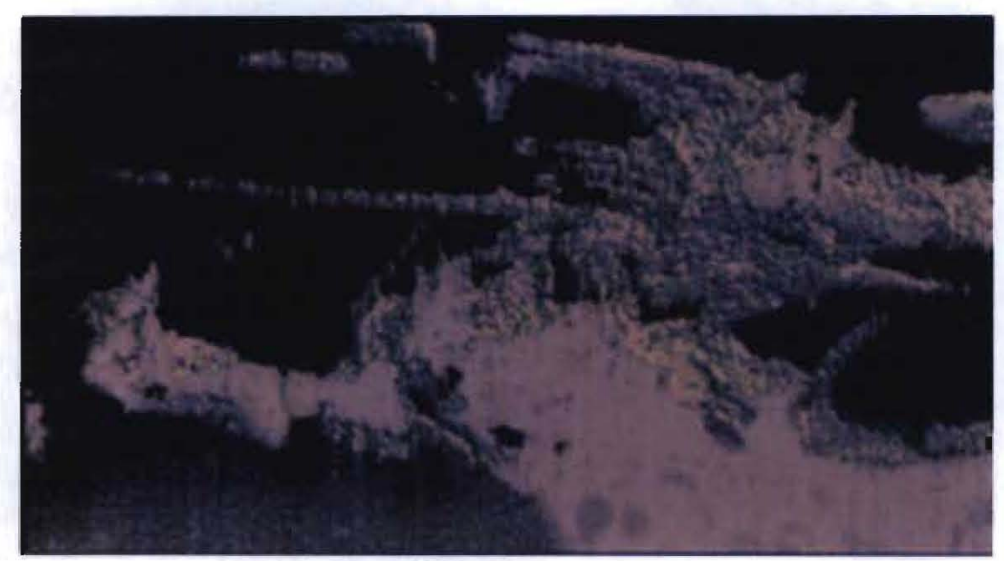

\section{$\mathbf{x}=\mathbf{0} \boldsymbol{m}$}

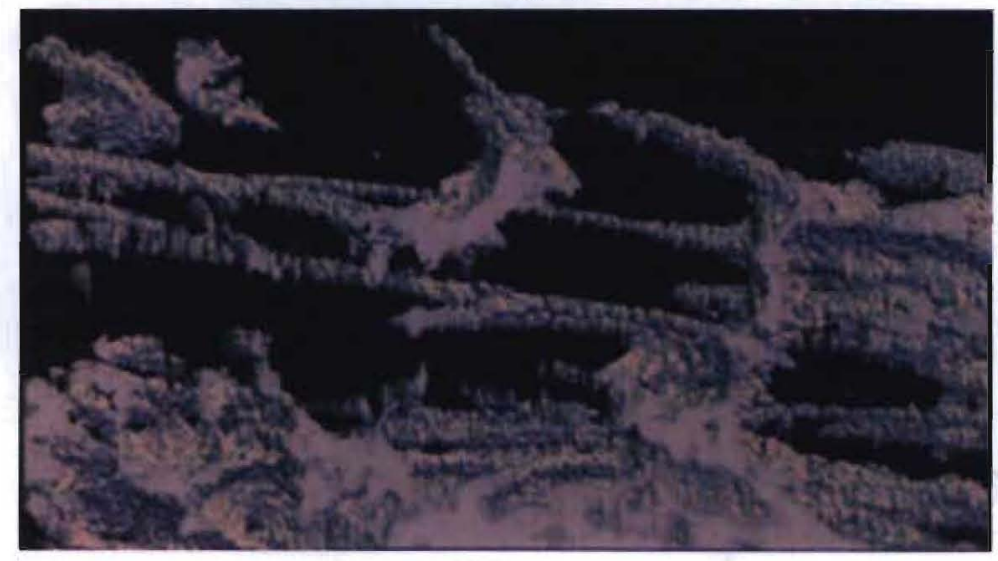

$\mathbf{X}=\mathbf{2 6 4 m}$

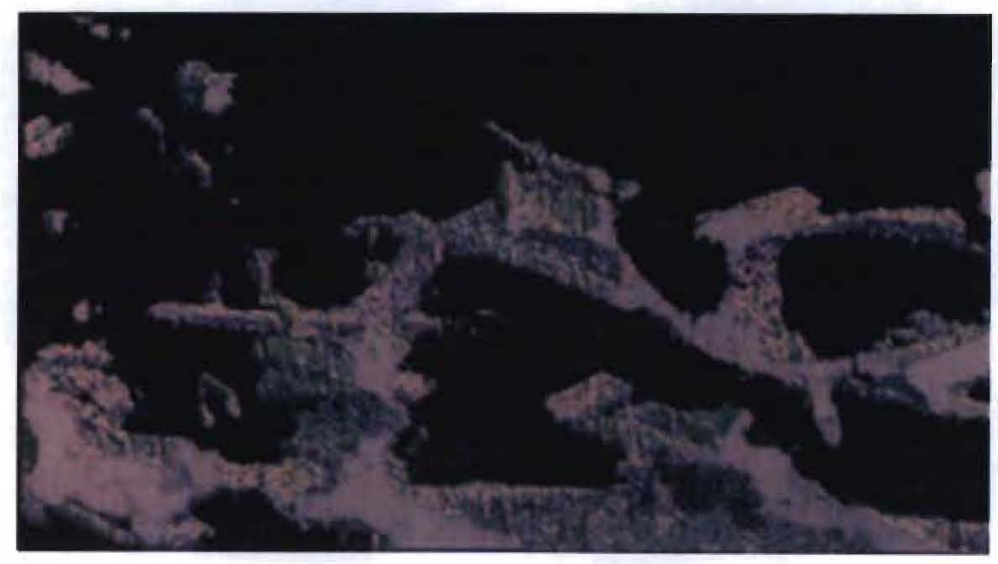

$\mathbf{X}=308 \mathrm{~mm}$

Figure 6: XY plane views of several 3-D thin sections along the $\mathrm{X}$ direction. 


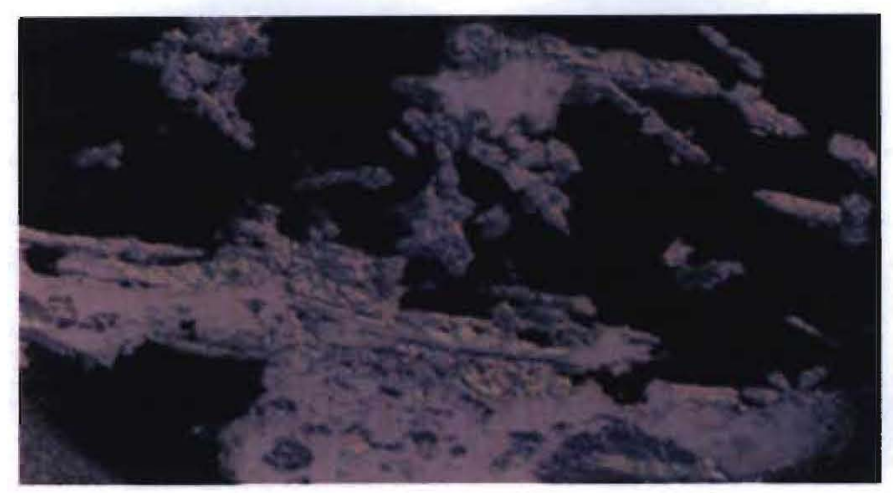

$z=44 \mathrm{~mm}$

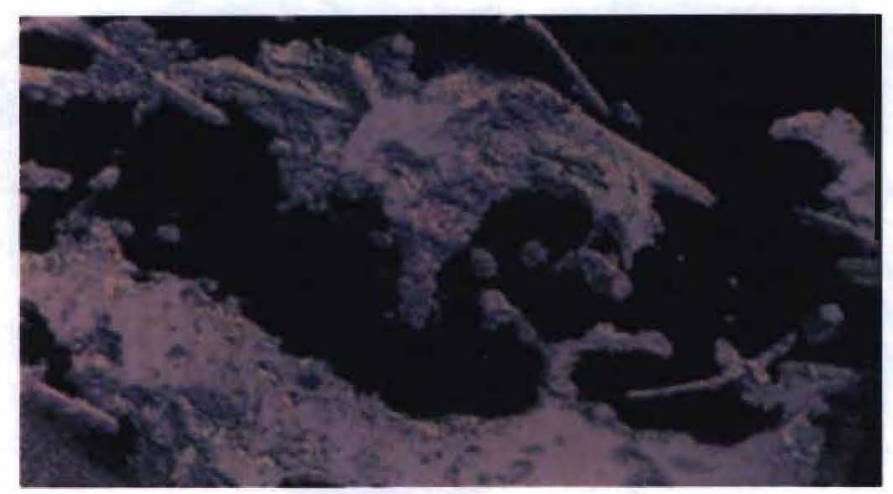

$\mathrm{Z}=176 \mathrm{~mm}$

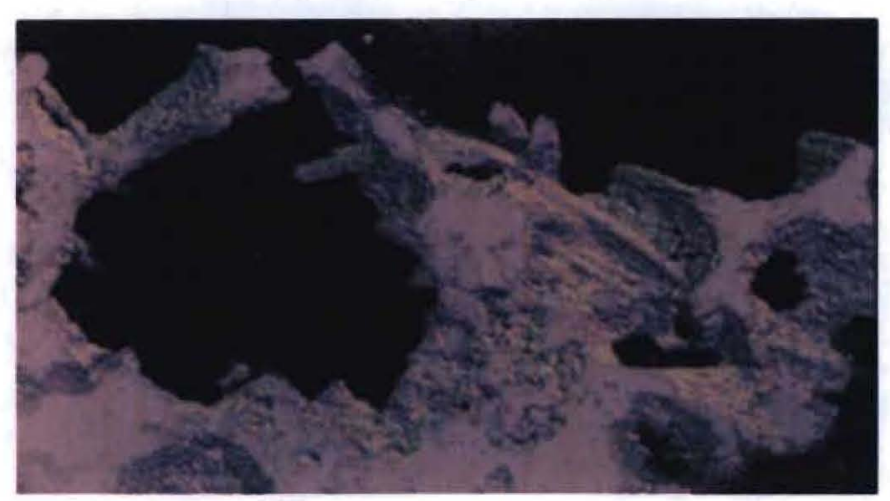

$2=308 \mathrm{um}$

Figure 7: YZ plane views of several 3-D thin sections along the $\mathrm{X}$ direction.

In order to investigate local porosity variations in the GDL sample, the solid volume fraction as a function of position was evaluated from the DVI dataset as the measure of the sample density and porosity. Solid volume fractions were measured by calculating the ratio between the number of voxels occupied (fiber + binder) and the total number of voxels in 3 -D volumes with $(50 \times 550 \times 50)$ voxles (i.e. $22 \mu \mathrm{m} \times 242 \mu \mathrm{m} \times 22 \mu \mathrm{m}$ physical size). Since, porosity is (1-SVF), higher SVF implies lower porosity. Figure 8 shows the SVF map of the sample. The local SVF variation is significant, which in turn exhibits wide variation in the local porosity distribution. While the sample shows the existence of highly dense area (green color), areas with very low SVF (red color) suggest the formation of potential through pores. 


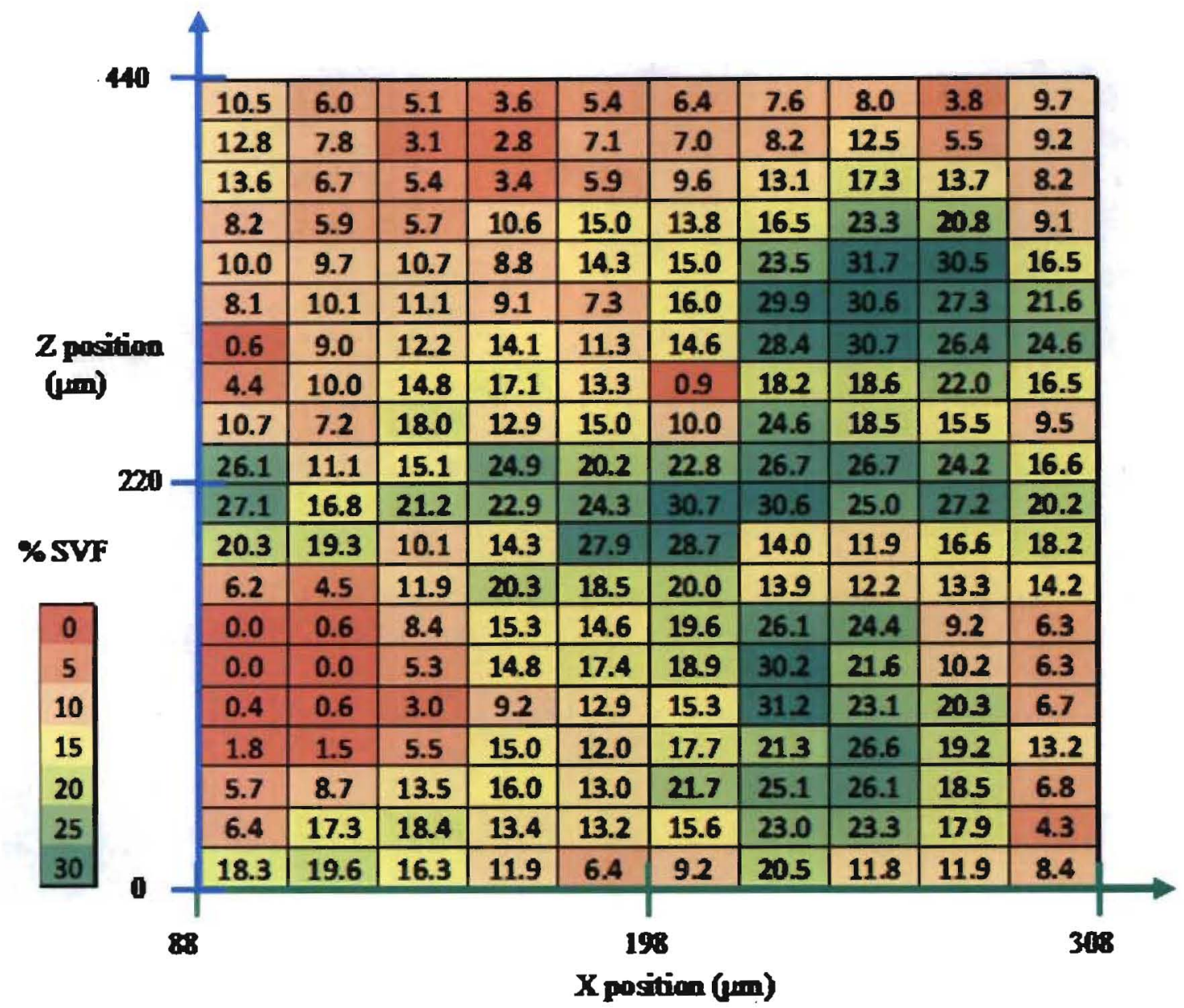

Figure 8: Solid volume fraction (SVF) map of the sample illustrating the local variation in porosity.

\section{Conclusions}

In this work, we reported our first attempt in 3-D microstructure reconstruction of the carbon paper GDL in a PEFC using the digital volumetric imaging (DVI) technique. The DVI, as opposed to the non-invasive X-Ray microtomography method, is a fluorescence technique based on automated serial sectioning of the sample impregnated with an embedding media. The 3-D thin sections obtained from the DVI method allow probing the internal pore morphology variation, fiber alignment and fiber/binder relative distribution in the sample. The reconstructed 3-D image along with the SEM image and the SVF map show significant variations in the local solid volume fraction and hence porosity in the through-plane and in-plane directions. The 3-D image obtained from the DVI technique is envisioned to provide meaningful microstructural information for inputs into high-fidelity direct numerical simulation methods for transport characterization and flooding behavior. Currently, efforts are underway to further investigate the internal pore structure and fiber alignment variations with different GDL samples using the DVI method. 


\section{Acknowledgments}

This work was supported by US Department of Energy, Energy Efficiency and Renewable Energy (EERE) Fuel Cell Technologies Program (Program Manager: Nancy Garland).

\section{References}

1. C. Y. Wang, Chem. Rev., (Washington, DC), 104, 4727 (2004).

2. R. L. Borup et al., Chem. Rev., (Washington, DC), 107, 3904 (2007).

3. P. P. Mukherjee, P. K. Sinha, and C. Y. Wang, J. Mater. Chem., 17, 3089 (2007).

4. P. P. Mukherjee, C. Y. Wang, and Q. Kang, Electrochim. Acta, 54, 6861 (2009).

5. P. P. Mukherjee, Q. Kang, R. Mukundan, and R. L. Borup, ECS Trans., 26, 97 (2010).

6. M. F. Mathias, J. Roth, J. Fleming, and W. Lehnert, in Handbook of Fuel Cells Fundamentals, Technology and Applications, W. Lietsich, A. Lamm and H. A. Gasteiger, Editors, Vol.3, Ch. 42, 517, John Wiley \& Sons, Chicester, 517 (2003).

7. V. P. Schulz, P. P. Mukherjee, J. Becker, A. Wiegmann, and C. Y. Wang, J. Electrochem. Soc., 154, B419 (2007).

8. R. Thiedmann, C. Hartnig, I. Manke, V. Schmidt, and W. Lehnert, J. Electrochem. Soc., 156, B1339 (2009).

9. M. Nelson, F. Garzon et al., $215^{\text {th }}$ ECS Meeting, San Francisco, CA, USA, May 24-29 (2009).

10. H. Ostadi, P. Rama, Y. Liu, R. Chen, X. Zhang, K. Jiang, Microelectronic Eng., 87, 1640 (2010).

11. H. Ostadi, P. Rama, Y. Liu, R. Chen, X. Zhang, K. Jiang, J. Membrane Sc., 351, 69 (2010).

12. J. Becker, V. P. Schulz, and A. Wiegmann, ASME J. Fuel Cell Sc. \& Tech., 5, 021006 (2008).

13. J. Becker, R. Flückiger, M. Reum, F. N. Büchi, F. Marone, and M. Stampanoni, J. Electrochem. Soc., 156, B1175 (2009).

14. V. Berejnov, D. Sinton, N. Djilali, J. Power Sources, 195, 1936 (2010).

15. D. Chinn, P. Ostendorp, M. Haugh, R. Kershmann, T. Kurfess, A. Claudet, T. Tucker, J. Manuf. Sci. Eng., 126, 813 (2004).

16. S. Jaganathan, H. V. Tafreshi, B. Pourdeyhimi, J. Colloid Interface Sci., 326, 166 (2008). 